\title{
Décrocheurs/raccrocheurs en Irlande
}

Comment interrogent-ils le système éducatif?

Responding to the needs of early school leavers and young people returning to education in Ireland

Alumnos que abandonan y que se reinsertan ¿cómo interrogan al sistema educativo irlandés?

\section{Scott Boldt}

Traducteur : Marion Latour

\section{CpenEdition} Journals

Édition électronique

URL : https://journals.openedition.org/ries/1724

DOI : $10.4000 /$ ries. 1724

ISSN : 2261-4265

Éditeur

France Education international

Édition imprimée

Date de publication : 1 avril 2004

Pagination : 67-80

ISBN : 978-2-85420-560-2

ISSN : $1254-4590$

Référence électronique

Scott Boldt, « Décrocheurs/raccrocheurs en Irlande », Revue internationale d'éducation de Sèvres [En ligne], 35 | avril 2004, mis en ligne le 23 novembre 2011, consulté le 08 juillet 2021. URL : http:// journals.openedition.org/ries/1724; DOI : https://doi.org/10.4000/ries.1724 


\section{Décrocheurs/ \\ raccrocheurs en Irlande}

Comment interrogent-ils le système éducatif?*

\section{Scott Boldt}

L'objectif de cet article est de décrire, de manière générale, la réponse apportée par le système éducatif de la République d'Irlande aux jeunes qui quittent la scolarité obligatoire avant d'atteindre l'âge légal (c'est-à-dire seize ans). Il convient de remarquer que le décrochage scolaire devient «problématique» tout d'abord lorsque ceux qui quittent l'école de manière anticipée ne trouvent pas ou ne parviennent pas à trouver une formation professionnelle ou un emploi. Ce problème devient d'autant plus préoccupant lorsque, dans une société, ce sont les compétences acquises durant la scolarité qui déterminent l'entrée dans la vie active, l'avancement professionnel et le salaire.

Indépendamment de ce constat, le décrochage scolaire - le fait de quitter l'école avant la fin de la scolarité obligatoire - est un problème difficile; un certain nombre d'orientations politiques et d'initiatives ont été prises pour le traiter, de même que plusieurs programmes et réformes ont été mis en œuvre pour améliorer la situation.

\section{Contexte historique}

En Irlande, la montée du nationalisme et le conflit avec l'Angleterre a abouti en 1922 à un accord prévoyant la création de l'État libre d'Irlande et de l'Irlande du Nord. En 1949, la République d'Irlande était établie en tant qu'État; l'Irlande du Nord restait rattachée au Royaume-Uni. Cet épisode de l'histoire a eu un impact fort puisque, depuis l'indépendance, l'accent a été mis sur l'identité nationale et le renouveau de la langue. Le bilinguisme constitua dès 1922 un objectif prioritaire pour le ministère de l'Éducation.

Historiquement, les Églises irlandaises ont joué un rôle significatif en pourvoyant aux besoins d'instruction et d'éducation du pays. Le propre du système éducatif irlandais a toujours été d'être fortement marqué par les valeurs et les objectifs de l'église catholique. Même si cette influence a diminué ces dernières années, «au niveau de l'enseignement primaire, la grande majorité des établissements sont sous contrôle et gestion confessionnels. Tel est le cas des écoles secondaires ${ }^{1}$ qui appartiennent majoritairement aux autorités diocé-

\footnotetext{
* Article traduit par Marion Latour.

1. Ndt: appelées secondary schools ou voluntary secondary schools, ces écoles secondaires accueillent les enfants entre douze et dix-huit ans et représentent $56 \%$ des établissements de l'enseignement secondaire, qui compte également des écoles professionnelles, des écoles communautaires et des établissements polyvalents. Source: Structures of education, vocational training and adult education in Europe: Ireland, 2003, Eurydice.
} 
saines ou aux ordres religieux. Un nombre restreint d'écoles appartient à des laïcs chargés également de leur gestion ${ }^{2}$.

Ce n'est qu'à la fin $\mathrm{du} \mathrm{XX}^{\mathrm{e}}$ siècle que l'Irlande a vu naître un véritable réseau d'écoles et un système éducatif formalisé, apte à pourvoir aux besoins d'une part importante de la population. «Il est communément reconnu que du début de l'Indépendance jusqu'à 1960, le système faisait preuve d'inertie et manquait sérieusement de ressources. Pendant la scolarité obligatoire, il y avait trop d'élèves par classe et le curriculum était beaucoup trop limité. À la rentrée scolaire de 1957, $22 \%$ des enseignants n'avaient pas reçu de formation. Le second cycle du secondaire était restreint, payant et quasiment réservé aux enfants issus des milieux les plus riches. L'enseignement supérieur était élitiste ${ }^{3}$.

Le rapport du ministère de l'Éducation de 1965 intitulé Investment in education ${ }^{4}$ affichait deux objectifs prioritaires: satisfaire les besoins d'une «économie en développement» et promouvoir «l'égalité des chances». En 1967, la gratuité fut introduite pour l'enseignement secondaire, ce qui entraîna un développement très important du secondaire et de l'enseignement professionnel. Cet accroissement du nombre d'élèves scolarisés engendra une prise de conscience de la nécessité de relever le défi que posait à un système historiquement rigide l'accueil de tous ces élèves.

Le rapport Investment in education soulignait très nettement «la nécessité de corriger le déséquilibre manifeste entre le rendement classique des écoles, d'une part, et les besoins d'une économie qui se développait à grande vitesse, d'autre part». Mais le rapport insistait presque autant sur l'égalité des chances, dont le bienfait était clairement reconnu par la communauté internationale, alors qu'en Irlande les disparités manifestes qui existaient entre classes sociales et entre régions dans la manière de pourvoir aux besoins éducatifs, allaient à l'encontre de ce principe d'égalité ${ }^{5}$.

«Depuis les années soixante, l'objectif de la scolarisation a évolué; ce changement transparaît dans les documents de politique éducative. Dans les années soixante-dix et quatre-vingts, le rôle que joue l'école dans le rétablissement de la langue et de la culture nationales a commencé à perdre du terrain, au profit d'impératifs de plus en plus économiques. Les écoles étaient exhortées à préparer les élèves à devenir des citoyens constructifs et actifs, à participer à la force de travail et à créer de la richesse pour elles-mêmes et pour les autres ${ }^{6}$.

Tout au long des années soixante-dix et quatre-vingts, des modifications ont été apportées au curriculum, à la fois pour les niveaux du primaire et du secondaire. "Cependant, le manque de coordination et le fait de ne pas avoir

\footnotetext{
2. Hyland in Furlong and Monahan ed., 2000, p. 23.

3. OCDE, 1991, p. 25.

4. Investment in education: Investir dans l'éducation.

5. Hannan, 1991, pp. 17-18.

6. Hyland, p. 26.
} 
réussi à planifier un programme éducatif en étapes progressives correspondant à l'âge et aux capacités intellectuelles, dès le niveau primaire, posèrent des problèmes considérables aux élèves et aux enseignants». En outre, l'extension et la diversification des réformes du curriculum dans les établissements du secondaire ont été freinées par une absence de vraie réforme du mode de certification officielle ${ }^{7}$. La plupart des développements essentiels du curriculum irlandais, au cours des années quatre-vingt-dix, furent issus de programmes locaux conçus dans les années soixante-dix et quatre-vingts. Ces innovations furent à l'origine de bien des évolutions du curriculum ces dix-huit dernières années.

\section{DÉVELOPPEMENT DU CURRICULUM}

Dans le système éducatif irlandais, les écoles jouissent individuellement d'une grande autonomie. Bien que les écoles suivent le curriculum national et que de nombreux aspects du système éducatif soient gérés et conçus au niveau central, les écoles disposent d'une grande marge de liberté. Dans une large mesure, ce sont les écoles qui déterminent leurs missions, leur politique d'admission, leur emploi du temps, leur personnel et leurs choix en termes de programme. «Au primaire, les écoles ont la liberté d'interpréter le programme national à l'intérieur d'un environnement personnalisé de Whole School Planning ${ }^{8}$. Il en va de même pour le secondaire ${ }^{9}$.

Cette latitude officielle a un revers: elle peut être perçue comme une absence de plan national global et coordonné, ce qui, par contrecoup, risque de limiter l'action et l'efficacité de la politique gouvernementale.

«Pour des raisons historiques, les écoles irlandaises jouissent d'une grande autonomie dans leurs activités quotidiennes, en particulier au niveau du secondaire. Dans la mesure où la plupart de ces établissements se trouvent dans des circonscriptions où cohabitent plusieurs écoles, celles-ci se trouvent en concurrence directe pour recruter les élèves. Au sein de la plupart de ces communautés, une certaine disparité entre écoles a implicitement émergé dans la manière de dispenser le programme, et de façon significative, dans leur public, en terme de sexe, d'aptitudes scolaires et de statut social. Bien que l'évolution historique du système explique ces disparités, l'une des conséquences les plus graves est l'absence, au niveau du primaire et du secondaire, d'une agence locale ou nationale qui puisse assurer que ce système scolaire local serve l'intérêt général ${ }^{10}$.

7. Coolahan, 1991, p. 135.

8. NdT: inaugurée en 1999, l'initiative School Planning ou School Development Planning est un processus de planification de l'éducation, dans lequel s'investissent tous les membres de la communauté scolaire, afin d'assurer à chaque élève une éducation de qualité. Pour plus d'informations: http://www.sdpi.ie/

9. Hyland, p. 27.

10. Coolahan ed., 1994, p. 107. 
Le côté positif de la liberté dont bénéficient les écoles est que cela crée des conditions favorables à l'innovation et au développement.

«Alors que le curriculum officiel a mis du temps à répondre à la plus grande diversité des élèves en termes d'aptitudes et de potentiels - conséquence de l'expansion du système - des initiatives locales de développements du curriculum se sont progressivement imposées. Dans les années quatre-vingt-dix, les nouveaux cours qui ont finalement été formalisés à l'échelle nationale... ont tous pour origine des développements inaugurés sur le terrain, qui remontent aux années soixante-dix et quatre-vingts ${ }^{11}$.

Tout au long des années quatre-vingt-dix, de gros progrès ont été faits pour aligner la politique des programmes d'études sur les meilleures pratiques éducatives et les besoins d'une population d'élèves diversifiée. Après de nombreuses années de développement et de planification qui ont donné lieu à une large consultation, on a institué en 1999 un curriculum au niveau de l'enseignement primaire. "Le curriculum est représentatif du courant de pensée éducatif du moment; il intègre les pratiques pédagogiques les plus innovantes et les plus efficaces pour satisfaire les besoins actuels des enfants irlandais». La phrase inaugurale du curriculum donne le ton; elle est évocatrice de l'esprit du programme. «Le curriculum de l'école primaire valorise l'unicité de l'enfant, tel qu'il s'exprime dans la personnalité, l'intelligence et le potentiel de développement de chacun ${ }^{12}$.

Au niveau du secondaire, le Junior Certificate ${ }^{13}$, introduit en 1989, est en cours d'extension et d'amélioration avec le Junior Cycle Schools Programme, le programme des écoles du premier cycle, qui permet d'adapter le curriculum aux besoins particuliers de certains élèves. Dans le cycle supérieur, on relève de nombreux développements et innovations, dont la Transition Year ${ }^{14}$, le Leaving Certificate Vocational, le Leaving Certificate Applied, ainsi que des réformes portant sur les disciplines et les pratiques du Leaving Certificate ${ }^{15}$ général.

«Ces certificats ont tous été conçus pour répondre aux aptitudes et aux intérêts de plusieurs catégories d'élèves. Des changements sont également survenus dans l'organisation des disciplines et l'introduction de nouvelles matières telles que l'instruction civique, sociale et politique et l'éducation sociale, personnelle et sanitaire, qui ont une résonance particulière au regard de la culture et du comportement scolaires. Des efforts ont été faits pour diversifier les modes d'apprentissage - enseignement, avec une importance plus grande accordée à

\footnotetext{
11. Hyland, p. 25.

12. Coolahan in Furlong and Monahan ed., 2000, p. 117.

13. Certificat d'études du premier cycle de l'enseignement secondaire, que les élèves passent à l'âge de quinze ans. 14. NdT: introduite à la rentrée 1994, cette année facultative assure la transition entre les deux cycles de l'enseignement secondaire. Actuellement, $40 \%$ des élèves suivent ce programme. Source: Structures of education, vocational training and adult education in Europe: Ireland, 2003, Eurydice.

15. NdT: le Leaving Certificate est le diplôme de fin d'études secondaires, que les élèves passent vers dix-huit ans. Il se décline en Leaving certificate (général), en Leaving certificate vocational (professionnel) et en Leaving certificate applied (appliqué).
} 
«l'apprentissage à apprendre. [...] Les efforts pour réformer les processus d'évaluation ont été moins concluants et ceci a également eu une incidence sur la culture des écoles » ${ }^{16}$.

Comme nous le verrons ultérieurement, ces développements qui ont touché le curriculum officiel sont en rapport avec notre sujet, car, à de multiples égards, ils s'efforcent de traiter le problème du décrochage scolaire et de prendre part à l'approche globale adoptée par le système éducatif irlandais vis-à-vis de cette question.

\section{Principes de la politique ÉDUCATIVE}

La politique éducative irlandaise favorise habituellement une approche holistique $^{17}$ de l'éducation pour tous les élèves. Selon Coolahan ${ }^{18}$, "l'une des caractéristiques les plus marquantes de notre système a été l'attention prêtée aux dimensions cognitives et spirituelles, mais aujourd'hui une plus grande attention est accordée à l'affectif, au social, à l'esthétique et au physique, dans le contexte d'une plus grande sensibilisation à l'intelligence multiple. En outre, il existe une plus grande prise de conscience du besoin qu'ont les élèves d'être guidés et conseillés. [...] La tradition selon laquelle l'enseignant est responsable du bien-être général de ses élèves a eu un impact très favorable, de même que le mouvement de promotion de l'hygiène par l'école, le Health Promoting School ${ }^{19}$ ".

Publié en 2000, le Plan de développement national pour l'Irlande 20 soulignait l'efficacité du "partenariat » comme garant du succès de l'économie et reconnaissait le rôle central de «la cohésion sociale» dans le soutien et la pérennité des accords de partenariat. L'intégration sociale est un objectif stratégique pour lequel des engagements spécifiques ont été pris.

Cinq principes-clefs ont étayé le Livre blanc sur l'éducation ${ }^{21}$ signé par le gouvernement en 1995 : égalité, pluralisme, partenariat, qualité, responsabilité. "Ces cinq principes découlent des objectifs fondamentaux que poursuit

16. Coolahan, 2000, p. 118.

17. NdT: de holisme, théorie selon laquelle l'homme est un tout indivisible qui ne peut être expliqué par ses différentes composantes (physique, physiologique, psychique) considérées séparément. Source : Le nouveau petit Robert.

18. Coolahan, 2000 , p. 118.

19. NdT: améliorer la qualité de l'éducation est l'une des priorités du ministère de l'Éducation d'Irlande du Nord, qui a formalisé cet objectif dans son programme intitulé "School Improvement: the Northern Ireland Programme». Parmi les missions que le programme définit pour l'école, la promotion de l'hygiène figure en bonne place. The Health Promoting School: a school self-assessment guide, guide conçu par l'Agence de l'Irlande du Nord pour la promotion de l'hygiène, en association avec le groupe de liaison de l'éducation à l'hygiène, est consultable sur : http://www.healthpromotionagency.org.uk/Resources/children/pdfs/HPSselfassess.pdf

20. NdT: lancé par le gouvernement irlandais pour la période 2000-2006, le National Development Plan for Ireland concerne des secteurs aussi variés que les services de santé, l'enseignement, les transports publics, les infrastructures routières, l'industrie, etc. Il représente un investissement de plus de 52 milliards d'euros provenant de fonds privés, publics et de subventions de l'Union Européenne. Site officiel: http://www.ndp.ie

21. Le White paper on education, définit les grandes orientations de la politique éducative, du préscolaire à la formation continue. Pour une présentation synthétique : http://www.trainersnetwork.org/html/policy18a.html 
l'éducation, à savoir favoriser le bien-être individuel, social et économique et améliorer la qualité de vie. Afin de remplir ces objectifs, le système éducatif irlandais maintient un équilibre délicat entre un contrôle au niveau central et une liberté au niveau des écoles ${ }^{22}$.

À de nombreux égards, le ministère de l'Éducation et de la Science travaille en partenariat avec les écoles (c'est-à-dire avec les organisations qui représentent respectivement la direction, les administrateurs, les principaux, les enseignants, les élèves, les parents et les collectivités). En outre, le ministère a récemment exhorté les écoles à conclure des partenariats avec les communautés extérieures à l'école et à clarifier le rôle joué par plusieurs partenaires intervenant dans le processus scolaire.

Rares sont les objectifs éducatifs qui peuvent être atteints sans la mise en place et le fonctionnement efficaces de partenariats appropriés. Il a été dit au sujet des politiques portant sur l'abandon scolaire «qu'il est fondamental que la lourde charge qui consiste à prendre à bras le corps ce problème, qui va en s'aggravant, ne soit pas laissée à la seule éducation, mais soit plus largement partagée par tout l'éventail de la politique gouvernementale». L'un des représentants du ministère s'exprimait en ces termes: «l'inégalité dans le domaine de l'éducation est l'un des aspects du problème plus large que représente l'inégalité socio-économique, elle-même un défi multiforme exigeant une solution multiforme, dont l'éducation fait partie. [...] La mobilisation des parents et de la communauté est devenue, depuis, un élément crucial ; ce processus a été encouragé par les Accords nationaux de partenariat ${ }^{23}$.

Il est vrai que le système éducatif ne peut, et ne doit pas, être tenu pour responsable des inégalités socio-économiques qui contribuent au problème du décrochage scolaire; mais, en même temps, le facteur scolaire est déterminant pour traiter le problème. Cependant, les dispositions du système éducatif irlandais, en termes de structures et de politiques, sont telles que les stratégies et les objectifs doivent passer par le filtre des écoles. En même temps, en l'absence d'une stratégie et d'une vision pour traiter l'abandon précoce, de nombreux programmes et initiatives démarrent sur le terrain, puis nécessitent des efforts pour être reconnus et financés.

\section{Programmes de PRÉVENTION DE L'ABANDON SCOLAIRE}

En Irlande, l'essentiel de l'enseignement préscolaire a été pris en charge par des groupes privés et des particuliers qui dirigent des écoles maternelles, des garderies, des crèches, des groupes Montessori et des espaces de jeux. Bien que

22. Hyland, pp. 26-27.

23. O'Maolain in Dublin Employment Pact, 2002, p. 27. NdT: ces accords de partenariat à l'échelle nationale, les National Partnership Agreements, rassemblent le gouvernement, les syndicats et le patronat. Ils ont contribué à la reprise économique de l'Irlande à la fin des années quatre-vingts. 
la recherche irlandaise et internationale n'avait cessé d'insister sur l'importance des premières années de développement de l'enfant pour son parcours scolaire et sa réussite ultérieure, la politique éducative avait prêté une attention insuffisante au préscolaire, en termes de satisfaction des besoins et de financement.

Le besoin d'une meilleure prise en charge de l'enseignement préscolaire était, et demeure, considérable. Le Early Start Programme ${ }^{24}$, programme lance par le ministère de l'Éducation et de la Science, a en ce sens été une action clé. «L'objectif global que poursuit le programme préscolaire du ministère consiste à compenser les carences du milieu social ${ }^{25}$. D'un point de vue systémique, l'enseignement dans les premières années n'a pas été traité par le ministère dans le cadre d'une politique sérieuse ni par une approche stratégique digne de ce nom pour traiter de l'échec et du décrochage scolaires.

Au niveau du primaire, on a toujours accordé une grande importance au développement holistique de l'enfant et on a pris de plus en plus conscience du besoin d'aider les enfants qui manifestent des signes associés à l'échec et à l'abandon scolaires. Les initiatives et les approches à ce niveau sont variées et impliquent les composantes suivantes: livres et repas scolaires ciblés, cours de soutien, mesures particulières pour les écoles considérées comme «désavantagées » et engagement des parents.

Un nouveau programme pour les élèves du primaire issus des milieux défavorisés, le New Programme for Primary Pupils from Disadvantaged Backgrounds, a englobé d'autres initiatives ministérielles ${ }^{26}$. Ce regroupement a paru nécessaire parce que l'on a considéré que ces programmes ne répondaient pas suffisamment, en termes d'accès, aux besoins d'une minorité significative de la population scolaire. Parallèlement à la conception d'un système subtil d'assistance et de mesures préventives pour les élèves à risque, les écoles devaient accepter d'identifier les besoins de ces enfants et d'y répondre, plutôt que compter sur les élèves pour s'adapter au programme officiel et à la structure en place. Il est encore trop tôt aujourd'hui pour estimer si ces dispositions seront comprises et mises en pratique avec succès.

L'une des initiatives les plus importantes, au niveau du primaire et du secondaire, a été le Home-School-Community Liaison Scheme ${ }^{27}$. Elle illustre parfai-

24. NdT: programme préscolaire pilote, financé par le ministère de l'Éducation et de la Science, en faveur des enfants ayant le moins de chances de succès dans leur scolarité.

25. Ministère de l'Éducation, 1995, p. 17.

26. NdT: il s'agit du Support Teacher Project et du Breaking the Cycle. Les support teachers, pour le soutien scolaire, viennent en aide aux enfants en difficulté qui souvent sont confrontés à des situations familiales compliquées ou pénibles: absentéisme des parents, pauvreté, etc. Le Breaking the Cycle est un programme pilote qui fut introduit en 1996 dans les écoles primaires pour venir en aide aux élèves les plus défavorisés, en particulier ceux dont les parents ont un faible niveau d'instruction. Source: Évaluation de l'Éducation pour tous 20oo: rapport pays, Unesco. http: //www2.unesco.org/wef/countryreports/ireland/contents.html

27. NdT: institué en 1990, ce programme qui assure la liaison entre la communauté, l'école et la famille, a pour mission de fédérer les compétences et expériences de ces différents acteurs, au service d'une meilleure qualité de l'éducation. Pour en savoir plus: http://www.spd.dcu.ie/ed_dis_support/home_school_community.htm 
tement ce que peut être un programme, au départ local, qui est ensuite reconnu, puis étendu et enfin généralisé. "L’idée qui est au cœur de ce programme est de promouvoir une coopération active entre le foyer, l'école et les organismes appropriés de la communauté, pour susciter un intérêt des enfants pour l'éducation. Pour amener les parents à travailler en partenariat avec l'école, le programme vise à faire prendre conscience aux parents de leurs propres capacités à améliorer le processus éducatif de leurs enfants et à les aider à développer des compétences ${ }^{28}$.

Dans les écoles primaires, les enseignants spécialisés ${ }^{29}$ soutiennent certains élèves identifiés comme étant des enfants à risque. Les assistants qui interviennent en classe, à titre bénévole ou salarié, aident les enseignants; ils font partie des moyens mis à disposition des enfants qui rencontrent des difficultés au niveau du primaire.

En dehors des heures de cours, les «clubs de petit-déjeuner» et les «clubs de devoirs" sont largement institutionnalisés; ils ont souvent été mis en place de manière informelle, dans le but de soutenir les élèves à risque. Les structures de la communauté locale jouent elles aussi un rôle important - dans certains cas, elles sont les seules à intervenir en complément de l'école - en offrant un éventail de services aux élèves et aux parents.

Des programmes ont souvent été montés grâce au Home-SchoolCommunity Liaison Scheme pour aider les élèves à passer du primaire au secondaire. Cette transition est considérée comme un moment important du parcours scolaire, en particulier pour les élèves les plus fragiles. Les initiatives réussies d'aide à cette transition ont nécessité une coopération étroite entre les établissements, un programme d'accueil et d'intégration bien planifié, et un soutien continu des élèves.

Au niveau du secondaire, les interventions ciblées sur les élèves à risque sont moins nombreuses que dans le primaire. En revanche, en comparaison avec le primaire, il y a eu des développements plus importants au niveau du curriculum, au premier comme au second cycle. Bien que certains de ces développements aient déjà été mentionnés ci-dessus, le Leaving Certificate Applied ${ }^{30}$ mérite d'être signalé pour ses qualités innovantes.

Le Leaving Certificate ${ }^{31}$ est le diplôme qui sanctionne l'examen final, traditionnel et scolaire que les élèves passent à la fin de leur enseignement secondaire. C'est le certificat qui ouvre les portes de l'enseignement supérieur et, très souvent, de l'emploi.

\footnotetext{
28. Ryan in Boldt ed., 1996, p. 72.

29. Il s'agit des remedial teachers, pour les enfants socialement défavorisés, et des resource teachers, qui se consacrent aux enfants ayant des besoins spécifiques.

30. Voir note 15 .

31. Idem.
} 
Le Leaving Certificate Applied ${ }^{32}$ est un programme à part entière qui constitue une alternative au Leaving Certificate. Il repose sur un apprentissage pratique, un travail structuré en projets et un engagement actif de l'élève. Il est ouvert à tous mais s'adresse en priorité aux élèves qui ont peu de chances de réussir avec le programme traditionnel, ou ceux qui sont susceptibles d'abandonner l'école en cours de scolarité. Les élèves développent un éventail plus large de compétences et améliorent leurs capacités d'apprentissage. Dans un système d'enseignement secondaire aussi compétitif et qui accorde une place aussi importante à l'enseignement général, le Leaving Certificate Applied constitue une réussite remarquable en terme de curriculum. Le Programme des écoles du premier cycle ${ }^{33}$, reprend un grand nombre des principes du Leaving Certificate Applied et permet de modifier les programmes d'apprentissage des élèves au sein de la structure normale de la classe.

Deux initiatives du ministère de l'Éducation et de la Science ont été intégrées au School Completion Programme ${ }^{34}$ : le 8 to 15 Early School Leaver Initiative (ESLI) ${ }^{35}$ et le "Stay in School» Retention Initiative (SSRI) ${ }^{36}$. Le School Completion Programme est emblématique de la manière dont le système éducatif irlandais traite le problème du décrochage scolaire. D’une part, on reconnaît que l'intervention à l'école doit être fédérée et ciblée; et d'autre part, les écoles parties prenantes de ces initiatives ont bénéficié d'une grande marge de manœuvre pour concevoir des réponses et des projets et identifier des approches pertinentes qui ont pu ensuite être étendues à plus grande échelle.

Colm O’Maolain pose les bases du programme et les principes directeurs de la politique éducative décidée par le ministère. "L'intervention repose sur les principes suivants: l'identification des besoins individuels et leur prise en charge, la discrimination positive, les services holistiques intégrés, de même que la mobilisation des parents et leur formation. Les composantes de l'intervention sont notamment: le développement social et personnel, l'offre de services à la fois dans le cadre de l'école et à l'extérieur, le soutien aux devoirs, ainsi que les activités de loisir et le sport. Des programmes d'études et des méthodes d'enseignement différenciés sont indispensables pour la stratégie définie et nécessitent de former le personnel. Un programme de parrainage impliquera le parrainage des plus jeunes par les élèves plus âgés, des élèves par les enseignants, et, enfin, des jeunes par des personnes importantes faisant partie de la communauté ou d'organisations professionnelles. Une approche holistique

32. Idem.

33. Junior Cycle Schools Programme.

34. NdT: programme dont l'objectif est d'optimiser la participation des élèves et leur présence, pour les conduire à la fin de la scolarité obligatoire. Pour plus d'informations: http://www.ncge.ie/service.asp?id=ED170070803

35. 8 to 15 Early School Leaver Initiative (ESLI): programme pour ceux qui quittent l'école entre huit et quinze ans.

36. "Stay in School» Retention Initiative (SSRI): programme «restez à l'école» pour garder les élèves à l'école. 
nécessite de développer les liens qui existent entre les enfants, entre les familles et les enfants, et entre les familles et la communauté ${ }^{37}$.

Cependant, le caractère autonome des écoles signifie que les approches qui soutiennent les élèves à risque et qui les gardent à l'école sont largement mal connues alors qu'elles ont fait leurs preuves. Enseignants, conseillers d'orientation, enseignants spécialisés ${ }^{38}$, professeurs principaux, tuteurs de classe, enseignants membre du Home-School-Community Liaison, personnel de la direction et aumôniers scolaires, tous peuvent s'investir pour traiter le décrochage scolaire, en jouant des rôles-clés et diversifiés à l'échelle de leur école. Néanmoins, il n'existe aucune approche systématique et les réponses apportées par les écoles dépendent essentiellement de leurs priorités locales et des ressources dont elles disposent. Personne, au sein du personnel de l'école, n'est mandaté pour surveiller les élèves à risque. En outre, il n'y a pas d'incitation du système éducatif pour assurer le suivi des élèves susceptibles d'abandonner l'école en cours de scolarité.

Aujourd'hui, avec la mise en œuvre du système des Education Welfare Boards ${ }^{39}$, l'ancien système des School Attendance Officers ${ }^{40}$, qui existait exclusivement dans les zones urbaines, a été aboli. Ces fonctionnaires sont censés être remplacés par les assistantes sociales scolaires ${ }^{41}$. Malheureusement, il faut regretter que ce service n'ait pas fonctionné. Vient s'ajouter à cela le fait que la légitimité dont bénéficiaient les policiers pour exiger la présence aux cours leur a été retirée.

Il est prévu que si un élève s'absente pendant vingt jours, l'école le signale à l'Education Welfare Board. Un agent EWO doit assurer le suivi en consultant ses parents. Depuis 2002, en l'absence d'un système en place, plus aucun service n'assure en Irlande une mission de soutien et de garantie de présence à l'école. C'est pourquoi le décrochage et l'échec scolaires sont provisoirement privés d'un outil essentiel. Cela a non seulement une incidence sur la situation individuelle de milliers d'élèves, mais pose également la question de savoir comment les données sur la présence scolaire et l'abandon des études en cours de scolarité sont gérées au plan national.

\section{INITIATIVES DE RACCROCHAGE}

Les ateliers de formation qui s'adressent aux jeunes ayant décroché et à la communauté constituent la principale réponse officielle donnée aux jeunes qui quittent l'école précocement sans avoir obtenu de qualifications réelles. Ces ateliers dispensent au maximum deux années d'enseignement, de formation et

37. O'Maolain, 2002, p. 28.

38. Il s'agit, entre autres, des resource teachers.

39. NdT: en 2000, la loi intitulée Education Welfare Act définit un cadre pour combattre l'absentéisme scolaire. Elle établit dans tout le pays des équipes d'assistantes sociales scolaires, les Education Welfare Boards.

40. NdT: fonctionnaires de l'Éducation chargés de veiller à la présence des élèves à l'école.

41. Education Welfare Officers (EWO). 
d'expérience professionnelle. L'accent est mis sur le développement de la sociabilité et de la capacité d'adaptation, tout en préparant progressivement les jeunes au monde du travail. Les principes et les approches adoptés s'apparentent à ceux décrits plus loin.

L'auteur de cet article ayant pris part, pendant un certain nombre d'années, à la recherche et à la diffusion des réponses apportées aux besoins de ceux qui abandonnent l'école en Irlande, il proposera une description générale de ces solutions. S'inscrivant dans des contextes spécifiques, ces initiatives sont diverses, c'est la raison pour laquelle il n'est pas possible de les décrire en détail ici; mais il est intéressant de souligner qu'elles se ressemblent à de nombreux égards.

Les projets qui ont été mis en place pour les jeunes ayant quitté l'école précocement sont généralement conçus en réponse à des besoins identifiables. Que le système éducatif officiel ne satisfasse pas ces besoins de manière adéquate et qu'une approche non scolaire soit nécessaire ont fait l'objet d'une prise de conscience. Les mesures d'aide développées sont conçues au niveau local.

La caractéristique de ces initiatives est qu'elles s'attachent avant tout et en priorité au développement des relations avec les jeunes. En général, elles suscitent l'adhésion des jeunes car elles sont à l'écoute de leurs besoins et les placent au centre des programmes. Elles n'adoptent généralement pas une approche normative; mais des limites sont clairement établies, à l'intérieur desquelles les jeunes et l'équipe travaillent ensemble, prennent les décisions et s'orientent vers des objectifs communs.

On insiste davantage sur le processus d'apprentissage que sur l'instruction et l'enseignement. Priorité est donnée à l'amélioration de la confiance personnelle et de l'estime de soi. Le soutien et/ou la mobilisation des parents et de la communauté locale au programme contribue de manière significative à son succès. Là où les initiatives sont centrées spécifiquement sur l'apprentissage, elles reposent sur des activités offrant aux jeunes une palette de possibilités et de choix. Enfin, les initiatives qui ont rencontré du succès sont celles qui ont été bien gérées; cependant, elles s'appuient souvent sur des bénévoles, un engagement personnel fort de la part de l'équipe, et toutes semblent sans cesse avoir à chercher des sources de financement.

Les actions en faveur des jeunes et de la communauté ont visé à proposer des expériences d'apprentissage non officielles et pragmatiques à la fois, pour contribuer au développement social et à l'épanouissement personnel des jeunes. Les personnels chargés de la jeunesse réussissent à entrer en contact avec les jeunes qui ont été exclus de l'école ou qui n'étaient pas capables de faire face à ses exigences. Le système éducatif officiel a beaucoup à apprendre des méthodes de travail que les services pour la jeunesse ${ }^{42}$ emploient avec les jeunes. 
Par ailleurs, les services pour la jeunesse contribuent largement à répondre aux attentes de ceux qui ont décroché.

«Les services pour la jeunesse se sont engagés à donner des moyens et des ressources aux jeunes qui n'ont pas profité du système éducatif officiel, ou dont les besoins n'ont pas été satisfaits par ce système. Il s'agit d'une expérience éducative planifiée et systématique qui complète le curriculum officiel de l'école. (...) La plupart des organisations de jeunesse bénévoles répondent aux besoins des jeunes défavorisés. (...) Ces organisations développent des services de prévention qui s'adressent plus particulièrement aux jeunes à risque ${ }^{43}$.

Les définitions de l'abandon en cours de scolarité, et par voie de conséquence, les données correspondantes, sont variables. À partir des études menées par les chercheurs et des statistiques du gouvernement, on estime que plus de $15 \%$ de la population peuvent être considérés comme défavorisés sur le plan scolaire (il s'agit des personnes ayant quitté l'école sans qualification formelle ou de celles n'ayant pas réussi à obtenir cinq mentions "passable» au Junior Cerficate $\left.{ }^{44}\right)$. Il est intéressant de remarquer que la majorité de cette population vit en zone rurale $(60 \%)$, et $26 \%$ à Dublin ${ }^{45}$.

À la lumière de son développement historique et des disparités socioéconomiques qui existent dans la société irlandaise, force est de constater que le système éducatif a fait de sérieux progrès - réels et qu'il faut saluer - en termes de services rendus à la population et d'offre scolaire. L'Irlande a répondu aux besoins des jeunes qui connaissent l'échec scolaire et qui risquent de quitter, ou ont quitté, l'école avant l'âge légal de seize ans.

Les données relatives aux jeunes ayant quitté l'école sans qualification ont des facettes trop multiples pour être réduites à des causes précises appelant des solutions toutes faites. Plutôt que des solutions, ce sont des réponses qui conviennent pour traiter le problème. Ces réponses devront être variées, souples et évolutives.

En Irlande, ce qui est le plus encourageant ces vingt dernières années, c'est la prise de conscience du problème, la perception de sa complexité et l'engagement pris pour le traiter. Ceux qui décident des politiques à mener, les représentants de l'institution scolaire, les enseignants, les employeurs, les personnes travaillant au service de la jeunesse et de la communauté, tous ont reconnu l'importance du problème et ont développé de nombreuses stratégies et approches pour prendre en charge le jeune.

43. National Youth Council of Ireland, 1996, p. 13.

44. Voir note 15.

45. Kellaghan, et al., 1995, p. 47. 
Il est difficile de séparer les réponses irlandaises au décrochage scolaire de la situation du pays et de son système éducatif. "Toute intervention, à l'échelle d'un pays, d'une région ou d'un certain nombre d'écoles, se situe dans un contexte unique. Dans quelle mesure, alors, les résultats d'une intervention comme celle-ci peuvent-ils globalement s'appliquer à d'autres situations? ${ }^{46}$. En dépit de cette spécificité, nous pensons que les leçons que l'on peut tirer de l'Irlande, ainsi qu'un grand nombre de ses approches et de ses innovations, peuvent être utilement adaptées à d'autres contextes.

On peut accorder plus facilement sa confiance à une approche qui s'appuie sur des principes partagés et qui fait écho à des expériences personnelles. À l'image de cet article, les idées empruntées ailleurs devront toujours être transposées dans la structure de pensée et la culture du traducteur et du lecteur. J'espère que cet article aura fourni une bonne vue d'ensemble et un bon aperçu de l'approche irlandaise de l'échec et du décrochage scolaires. L'Irlande a elle-même sans doute profité d'initiatives empruntées à d'autres pays, puis adaptées. Cet article contribuera, je l'espère, à traiter ce problème et à proposer d'autres façons d'en venir à bout.

\section{BibLIOGRAPHIE}

BOLDT S. (1994): Listening and Learning: A study of the experiences of early school leavers from the inner-city of Dublin. Dublin: MIE.

BOLDT S. (1996) : Primary School Interventions. Dublin : MIE.

BOLDT S. (1998): Showing the Way: Responses and approaches to the needs of students and early school leavers. Dublin: MIE.

COOLAHAN J. (1991): Irish Education: History and Structure. Dublin : IPA.

COOLAHAN J., ed. (1994): National Education Convention Report. Dublin : Convention Secretariat.

Dublin Employment Pact (2002): Preventing Early School Leaving: Conference outcomes and policy recommendations. Dublin: Dublin Employment Pact.

FURLONG C. and MONAHAN L., eds. (2000): School Culture and Ethos. Dublin : MIE.

HANNAN D. and SHORTALL S. (1991): The Quality of Their Education. Dublin : ESRI.

Ireland (1995): Charting our Education Future: White Paper on Education. Dublin: Stationery Office.

46. Morgan, 1998, p. 95. 
KELLAGHAN T., WEIR S., OHUALLACHAIN ••, and MORGAN M. (1995): Educational Disadvantage in Ireland. Dublin: Combat Poverty Agency.

MORGAN M. (1998): Early School Leaving Interventions: International comparisons. Dublin: Combat Poverty Agency.

National Youth Council of Ireland (1996): Submissions on Early School-Leavers and Youth Unemployment. Dublin: NESC.

OCDE/OECD (1991): Reviews of National Policies for Education: Ireland. Paris: OCDE/OECD.

Youthstart (1998): Prevention of Early School Leaving: Conference papers. Dublin: Youthstart. 